\title{
Subsequent anti-VEGF therapy after first-line anti-EGFR therapy improved overall survival of patients with metastatic colorectal cancer
}

This article was published in the following Dove Press journal:

OncoTargets and Therapy

\author{
Tianzhu Qiu ${ }^{1, *}$ \\ Wensen Chen ${ }^{2, *}$ \\ Ping $\mathrm{Li}^{\prime}$ \\ Jing Sun' \\ Yanhong Gu' \\ Xiaofeng Chen' \\ 'Department of Oncology, First \\ Affiliated Hospital of Nanjing Medical \\ University, Nanjing, People's Republic \\ of China; ${ }^{2}$ Infection Management \\ Office, First Affiliated Hospital of \\ Nanjing Medical University, Nanjing, \\ People's Republic of China \\ *These authors contributed equally \\ to this work
}

Correspondence: Xiaofeng Chen; Yanhong Gu

Department of Oncology, First Affiliated Hospital of Nanjing Medical University, 300 Guangzhou Road, Nanjing 210029,

People's Republic of China

Tel +86 I35 85I7 2066;

$+8613813908678$

Fax +862568217221

Email chenxiaofengnjmu@I63.com; guluer@163.com

\begin{abstract}
Colorectal cancer is one of the leading causes of cancer deaths worldwide. Due to targeted therapy, overall survival (OS) of metastatic colorectal cancer (mCRC) patients has been significantly increased over the past decade. However, the best sequencing of the therapeutic agents to be used in RAS wild-type subgroup is still under research. To determine the efficacy of targeted therapy, we collected randomized controlled trials which included patients receiving anti-epidermal growth factor receptor (EGFR) monoclonal antibody as first-line therapy in RAS/ KRAS wild-type mCRC. In our study, we found that OS was significantly improved by antivascular endothelial growth factor (VEGF) agent after first-line anti-EGFR therapy. Our results revealed that it is a sensible treatment strategy to try anti-VEGF agent after first-line combination therapy with anti-EGFR monoclonal antibody for RAS/KRAS wild-type mCRC.

Keywords: metastatic colorectal cancer, targeted therapy, overall survival
\end{abstract}

\section{Introduction}

Colorectal cancer (CRC) is one of the leading causes of cancer deaths worldwide. Before 2003, chemotherapy was extensively used for metastatic colorectal cancer (mCRC). Cytotoxic agents, especially irinotecan or oxaliplatin coupled with fluorouracil [FU], were proved to be effective in the treatment of $\mathrm{mCRC}$ and regarded as standard therapeutic regimens. ${ }^{1}$ Over recent years, targeted therapy has been shown to improve outcomes in patients with $\mathrm{mCRC}$ when added to chemotherapy regimens.

Targeted therapeutic drugs act on specific cellular pathways that drive tumor growth. The epidermal growth factor receptor (EGFR) is one of the 4 transmembrane growth factor receptor proteins and overexpressed in CRC cells. Stimulation of EGFR pathways could promote tumor cell motility, adhesion, and metastasis. ${ }^{2}$ Expression of EGFR has been associated with poor prognosis and decreased survival in patients with mCRC. Thus, anti-EGFR therapy is an important treatment option for these patients. Panitumumab and cetuximab are the 2 anti-EGFR monoclonal antibody drugs approved by the US Food and Drug Administration for the treatment of mCRC. In addition to EGFR-targeted therapies, vascular endothelial growth factor (VEGF)-targeted therapies have also proven clinical benefits in the treatment of patients with $\mathrm{mCRC}$ by inhibiting angiogenesis. ${ }^{3,4}$

RAS is a family of related proteins involved in transmitting signals within cells. RAS proteins are important downstream effectors in MAPK pathway that couples EGFR with intracellular signaling cascades. KRAS mutation is the most common RASmutated clone. KRAS mutations have been described in codons 12 and 13 (exon 2), 
and in rare cases, in codon 61 (exon 3). These mutations activate EGFR signaling pathway, making anti-EGFR therapeutic agents ineffective. ${ }^{5}$

In the last decade, many clinical trials have been implemented to assess the efficiency of targeted therapy in mCRC. The addition of cetuximab and FOLFIRI (irinotecan in combination with FU and leucovorin [LV]) as first-line treatment was used in both CRYSTAL and FIRE-3 studies. In CRYSTAL study, overall survival (OS) was only 23.5 months in KRAS wild-type and 28.4 months in RAS wild-type patients, which was shorter than that in FIRE-3 study (28.7 and 33.1 months, respectively). It is intriguing that these trials using targeted therapies as first-line therapy for mCRC have shown substantial variations in the reported median OS. We speculated that OS was related to the percentage of patients who received subsequent treatment and the percentage of patients treated with both antibodies. The percentage of patients who received second-line treatment was similar in CRYSTAL and FIRE-3 study (65\% vs 69\%). In FIRE-3 study, about $32 \%$ of patients received bevacizumab, an anti-VEGF agent, after they failed to respond to cetuximab; this proportion is more than that in CRYSTAL study. Similarly, both PRIME and PEAK trials evaluated the efficacy of panitumumab plus FOLFOX (oxaliplatin in combination with FU and LV) as initial treatment for mCRC. OS was much longer in PEAK study, while the first-line progression-free survival (PFS) was similar. The percentage of patients who received second-line treatment was similar in PRIME and PEAK study (58.8\% vs 63\%), but the secondline use of bevacizumab was different.

Based on the preliminary data, we analyzed the published trials to investigate the influence of targeted therapy on OS. We hope our results could help to make an evidence-based clinical decision of using targeted therapy in KRAS/RAS wild-type mCRC patients.

\section{Methods}

\section{Identification of eligible studies}

We systematically searched 2 search engines, the Cochrane Library and PubMed, from inception to July 15, 2017. We also performed handsearches of European Society for Medical Oncology (ESMO) and American Society of Clinical Oncology (ASCO) to identify any additional relevant trials. All relevant publications were reviewed, and duplications of articles from the 2 search strategies were eliminated. The search was conducted by 2 investigators (QTZ and $\mathrm{CXF}$ ). Any disagreements were resolved by consensus with involvement of a third author (CWS).

\section{Search strategy}

CENTRAL (the Cochrane Library):

\#1 (colorect* or colon* or rect* or anal* or anus* or intestin* or bowel*) near/3 (carcinom* or neoplas* or adenocarcinom* or cancer* or tumor* or tumour* or sarcom*)

\#2 MeSH descriptor: [Colorectal Neoplasms] explode all trees

\#3 (\#1 or \#2)

\#4 (epidermal growth factor or EGR or EGFR or ErbB-1 or HER1 or Cetuximab or Panitumumab)

\#5 MeSH descriptor: [Epidermal Growth Factor] explode all trees

\#6 MeSH descriptor: [Receptor, Epidermal Growth Factor] explode all trees

\#7 (\#4 or \#5 or \#6)

\#8 MeSH descriptor: [Neoplasm Metastasis] explode all trees

\#9 metasta* or stage 4 or stage IV

\#10 (\#8 or \#9)

\#11 (\#3 and \#7 and \#10)

PubMed:

\#1 colorectal neoplasms [MeSH Terms]

\#2 colorect or colon or rect or anal or anus or intestin or bowel [All Fields]

\#3 (\#1 or \#2)

\#4 Receptor, Epidermal Growth Factor [MeSH Terms]

\#5 epidermal growth factor or EGR or EGFR or ErbB-1 or

HER1 or Cetuximab or Panitumumab [All Fields]

\#6 (\#4 or \#5)

\#7 Clinical Study [ptyp]

\#8 (\#3 and \#6 and \#7)

\section{Inclusion criteria}

All human-associated studies were included if they met the following criteria: 1) involving patients receiving anti-EGFR monoclonal antibody in combination with chemotherapy as first-line therapy; 2) involving KRAS or RAS wild-type patients; 3) randomized controlled trials; and 4) the following data could be obtained or speculated: OS, PFS, the percentage of patients receiving both antibodies, and the percentage of patients treated with any subsequent therapy (including chemotherapy or targeted therapy).

\section{Data extraction and statistical analysis}

Two investigators (QTZ and CXF) extracted data independently and reached a consensus on all the items. For each study, the following information was collected: first author, publication year, first-line regimen, recruitment period, 
gene status, number of patients, OS, PFS, the percentage of patients receiving both antibodies, the percentage of patients treated with any subsequent therapy, and the percentage of patients receiving EGFR-targeted therapy beyond progressive disease (PD). All statistical analyses were conducted with Stata version 11.2 software.

\section{Results}

A total of 926 articles (all published) were identified from PubMed and the Cochrane Library. Among them, 873 duplicated and unrelated articles were excluded. Of the remaining 53 articles, 14 were excluded because the required data were unavailable. One ongoing study (TAILOR) reported in ESMO was included by handsearching. Finally, 9 clinical trials containing 11 subgroups were eligible for our study (Figure 1).

\section{Data synthesis and missing data statement}

Not all included trials reported complete number of patients with subsequent therapy. One author (QTZ) contacted researchers via email to request further information. Unfortunately, we failed to get the original data from these trials. The detailed characteristics of the 9 studies are presented in Table 1. Detailed description of each included trial is discussed next.

CRYSTAL study investigated the efficacy of FOLFIRI plus cetuximab in the initial treatment of mCRC. In this trial, number of patients, PFS, and OS were obtained from an article by Van Cutsem et al, published in $2011 .{ }^{6}$ The percentage of patients treated with any subsequent therapy and the percentage of patients receiving EGFR-targeted therapy beyond PD were obtained from the original article by Van Cutsem et al, published in 2009. ${ }^{7}$ The CRYSTAL study did

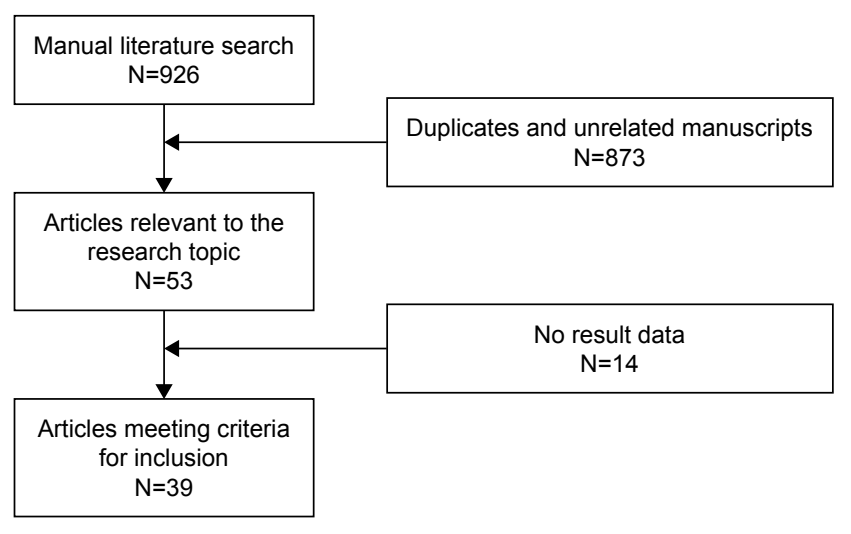

Figure I Flow diagram illustrating literature search and evaluation.

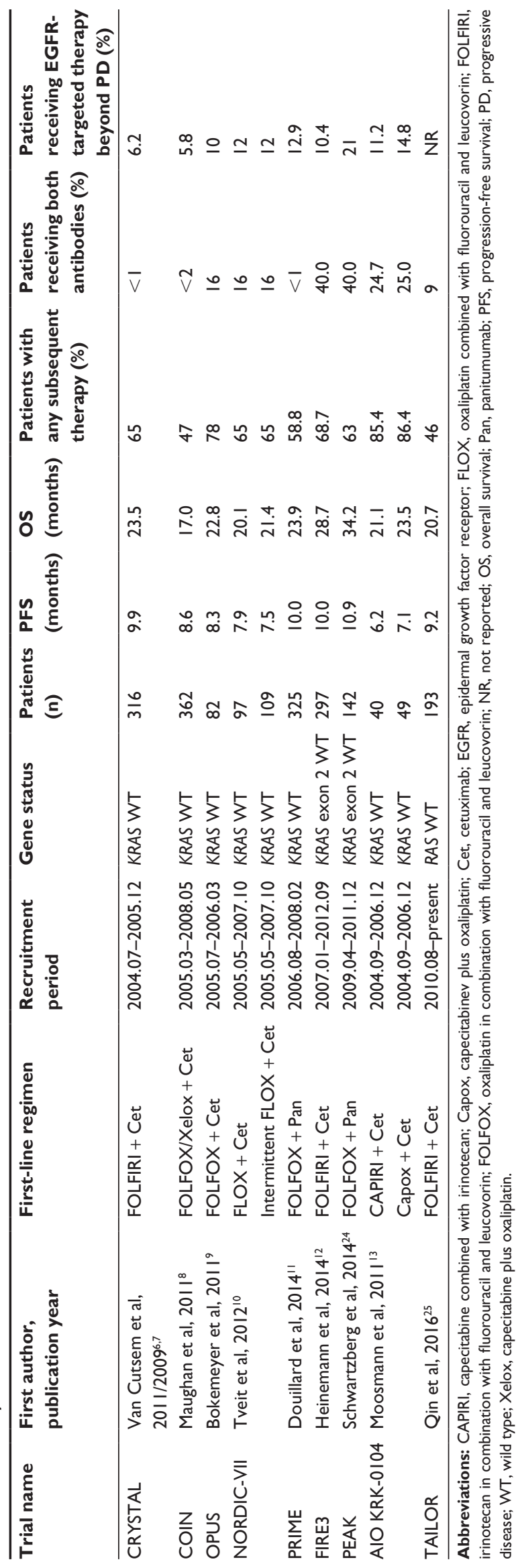


not report the number of patients who received bevacizumab; however, bevacizumab was approved by the European Union at the end of 2004, and we speculated that the use of bevacizumab in second-line treatment should be rare in 2004-2005, which was the recruit time for CRYSTAL study.

COIN trial assessed the effect on OS by adding cetuximab to oxaliplatin and fluoropyrimidine chemotherapy in arm B. Maughan et $\mathrm{al}^{8}$ reported that a total of 169 KRAS wild-type patients in arm B received any second-line therapy, among which 21 received EGFR-targeted therapy. Thus, the percentage of patients treated with any subsequent therapy was $47 \%$ (169/362), and the percentage of patients receiving EGFRtargeted therapy beyond PD was 5.8\% (21/362). COIN study did not report the number of patients who received bevacizumab. However, only 8 patients received other therapy except irinotecan, fluoropyrimidine, oxaliplatin, and EGFRtargeted therapy; thus, the percentage of patients receiving both antibodies should be $<2 \%(8 / 362)$.

OPUS study compared the efficacy of cetuximab plus FOLFOX-4 with FOLFOX-4 alone as first-line therapy for mCRC. Bokemeyer et al listed all poststudy anticancer treatments in Annals of Oncology. ${ }^{9}$ A total of 8 patients received EGFR-targeting agents (cetuximab), and 13 patients received VEGF-targeting agents (bevacizumab). Thus, the percentage of patients who received both antibodies was $16 \%(13 / 82)$, and the percentage of patients receiving EGFR-targeted therapy beyond PD was $10 \%$ (8/82). Though researchers listed all post-study anticancer treatments, they did not report the number of patients who received second-line therapy, because a patient could receive more than one of these agents. Since first-line regimen contained cetuximab, oxaliplatin, and 5-FU/folinic acid (FA), we speculated that bevacizumab, irinotecan, and other agents were used as second-line therapy by $78 \%(64 / 82)$ of patients. However, this is not an accurate data.

NORDIC-VII trial investigated the efficacy of cetuximab when added to bolus FU/FA and oxaliplatin (Nordic FLOX), administered continuously or intermittently, in previously untreated mCRC. The influence of KRAS mutation status on treatment outcome was also investigated. Tveit et al reported that second- or third-line treatment was administered in $67.3 \%$ of patients in KRAS wild-type group. ${ }^{10}$ We assumed that second- or third-line treatment was distributed equally in both arms, and thus, percentage of patients with any subsequent therapy was about $65 \%$. Tveit et al also reported that bevacizumab was administered in $17.0 \%$ and $15.0 \%$, and cetuximab in $12.4 \%$ and $11.2 \%$ of the patients in the 2 arms, respectively. We also used the data in KRAS wildtype population for analysis (the percentage of patients receiving both antibodies was about $16 \%$, and the percentage of patients receiving EGFR-targeted therapy beyond PD was about $12 \%$ ).

PRIME study evaluated the efficacy of panitumumab plus mFOLFOX as initial treatment for mCRC. In 2014, Douillard et al reported final results from PRIME in Annals of Oncology, ${ }^{11}$ in which they described that, in the KRAS wild-type subset, $12.9 \%$ of patients received antiEGFR-containing therapy and $58.8 \%$ of patients received chemotherapy. The percentage of patients with any subsequent therapy was replaced by the percentage of patients with chemotherapy for analysis $(58.8 \%)$. Since researchers did not report any VEGF-targeting therapy, we speculated that very few patients used bevacizumab $(<1 \%)$.

FIRE-3 trial was a randomized Phase III trial which compared the efficacy of FOLFIRI in combination with cetuximab or bevacizumab in patients with wild-type KRAS exon 2 tumors. Results of FIRE-3 were published in Lancet Oncology, ${ }^{12}$ in which Heinemann et al reported that secondline anticancer therapy was administered to 204 patients, bevacizumab was administered to 95 patients, and EGFR antibody was administered to 33 patients. Thus, the percentage of patients with any subsequent therapy was $68.7 \%(204 / 297)$, the percentage of patients receiving both antibodies was $31.99 \%$ $(95 / 297)$, and the percentage of patients receiving EGFR monoclonal antibody beyond PD was 10.4\% (33/362).

PEAK study compared panitumumab with bevacizumab while using mFOLFOX6 as the backbone chemotherapy. Subsequent anti-EGFR monoclonal antibody therapy was received by $21 \%$ of patients, and anti-VEGF therapy was received by $40 \%$ of patients. Similar to PRIME study, PEAK study only gave data of patients who received chemotherapy $(63 \%)$. This data was used as the percentage of patients with any subsequent therapy for analysis.

AIO KRK-0104 investigated the efficacy of cetuximab combined with capecitabine and irinotecan or capecitabine and oxaliplatin in the first-line treatment of mCRC. Similar to NORDIC-VII, Moosmann et al only reported secondline treatment in intention-to-treat population rather than in KRAS wild-type population. ${ }^{13}$

TAILOR is an open-label, randomized, multicenter Phase III trial that confirmed the efficacy of FOLFOX-4 plus cetuximab in the first-line treatment of Chinese patients with RAS wild-type mCRC. The results were first reported in ESMO World Congress on Gastrointestinal Cancer in 2016.

\section{Effects of interventions}

We analyzed the relationship between OS and the percentage of patients with any subsequent therapy, the percentage 


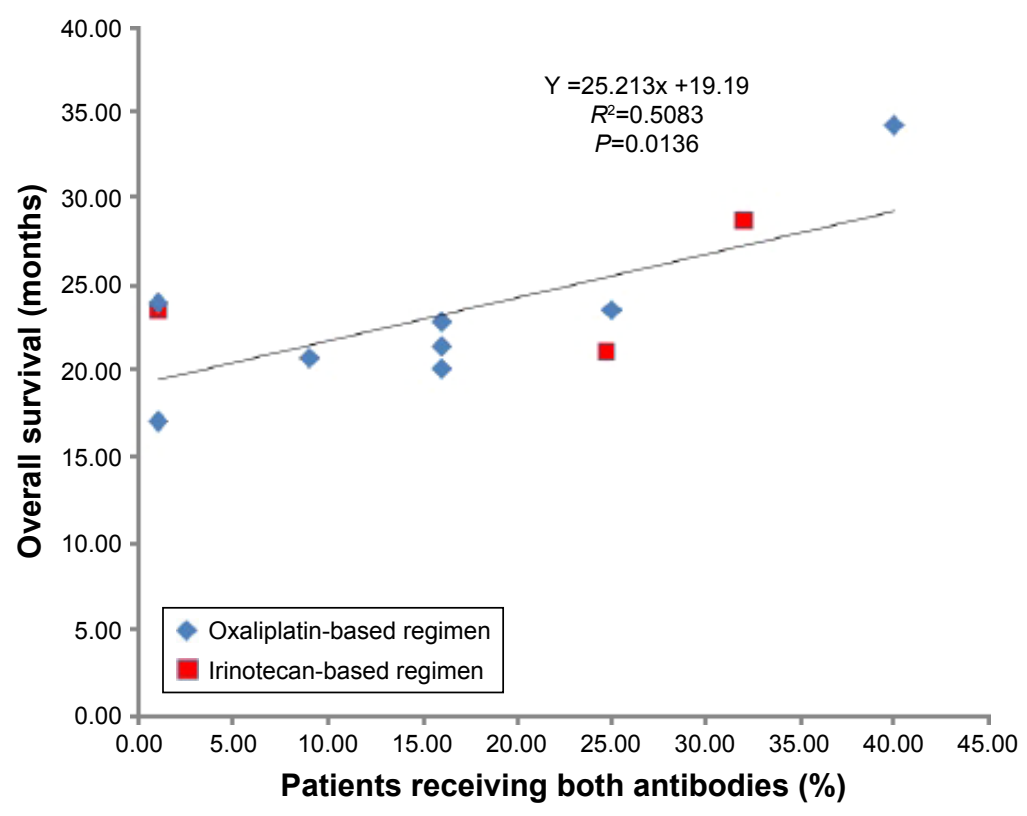

Figure 2 Regression plot and relationship between percentage of patients receiving both antibodies.

of patients receiving both antibodies, and the percentage of patients receiving EGFR-targeted therapy beyond PD. Figure 2 shows the relationship between OS and the percentage of patients receiving both antibodies. The results supported the idea that the OS was significantly affected by both targeted therapies $(P=0.0233)$. On the contrary, there is no correlation between OS and the percentage of patients with any subsequent therapy ( $P=0.8181$, data not shown) or the percentage of patients receiving EGFR-targeted therapy beyond PD ( $P=0.0722$, data not shown). Our results suggested that, after receiving anti-EGFR monoclonal antibody as first-line therapy, OS was correlated with subsequent availability of anti-VEGF therapy.

\section{Discussion}

Due to targeted therapy, the OS of mCRC patients has been significantly increased over the past decade, approaching about 30 months. A recent study has established that individuals with RAS wild-type mCRC have superior response to anti-EGFR monoclonal antibody therapy compared with RAS mutant subgroup. ${ }^{5}$ However, studies comparing the efficacy of first-line monoclonal antibodies in $\mathrm{mCRC}$ have yielded conflicting results, and the best sequencing of these agents in RAS wild-type subgroup is still under research. A recent meta-analysis reported that, compared with antiVEGF therapy, OS was improved by first-line anti-EGFR therapy in both KRAS wild-type and RAS wild-type patients, suggesting that anti-EGFR monoclonal antibodies may be a real alternative to anti-VEGF therapy as initial treatment for advanced CRC. ${ }^{14}$ Derangere et al found that VEGF-A could induce resistance toward cetuximab cytotoxicity on 3 KRAS and NRAS wild-type colon cancer cell lines, suggesting that a previous anti-VEGF therapy could decrease anti-EGFR efficacy. ${ }^{15}$ In our study, we found that after first-line antiEGFR therapy, OS was improved by subsequent anti-VEGF agents in patients with confirmed KRAS wild-type mCRC, which is consistent with the National Comprehensive Cancer Network (NCCN) guidelines.

However, there are still some shortcomings in our review. First of all, some studies were excluded because of incomplete data. For instance, CALGB/SWOG 80405 trial, ${ }^{16}$ a well-designed randomized Phase III study, was conducted to demonstrate a benefit for a cetuximab-containing regimen compared with a bevacizumab combination in KRAS exon 2 wild-type tumors. This trial has reported OS and PFS in cetuximab-containing first-line regimen. However, it did not give any information on subsequent treatment. Although we tried to contact researchers via email twice, we failed to get any information. Similarly, the CAIRO $2,{ }^{17} \mathrm{CECOG},{ }^{18}$ and PACCE ${ }^{19}$ studies were excluded for the same reason. We believe that conclusion would be more convincing if those trials were included.

In addition, most of the trials in our study reviewed patients with confirmed KRAS wild-type tumors instead of RAS wild-type tumors. In 2006, Lievre et $\mathrm{al}^{20}$ demonstrated that KRAS mutation is a predictor of resistance to cetuximab therapy and associated with a worse prognosis. However, a population of patients with KRAS wild-type tumors still did not respond to cetuximab; therefore, the influence of other downstream mutations on the efficacy of cetuximab 
was studied. De Roock et al found that BRAF and NRAS mutations were also significantly associated with a low response rate. ${ }^{21}$ More recently, in 2015, a meta-analysis revealed that $\sim 20 \%$ of KRAS exon 2 wild-type tumors harbored one of the new RAS mutations. Anti-EGFR therapy could improve both PFS and OS in patients harboring tumors without any RAS mutations, while no PFS or OS benefit was observed in those harboring tumors with the new RAS mutations. ${ }^{5}$

Furthermore, our review did not take primary tumor sidedness into account. Over recent years, we realized that primary tumors arising from the left and right sides of the colon have distinct clinical and molecular characteristics. CALGB/SWOG 80405 trial provided strongest evidence for the predictive value of primary tumor sidedness and response to EGFR inhibitors in the first-line treatment. OS was prolonged with cetuximab vs bevacizumab in the left-sided primary group but shortened in the right-sided primary group. In the 2017 NCCN guidelines, ${ }^{22}$ cetuximab or panitumumab was recommended for KRAS/NRAS wildtype and left-sided tumors only.

OS after first-line therapy was affected by regimens of subsequent chemotherapy or targeted therapy. As for chemotherapy, irinotecan and oxaliplatin alone or coupled with FU and LV are cornerstone drugs for patients diagnosed with advanced CRC. The same controversy of choosing the 2 kinds of cytotoxic agents happened in the period before targeted therapies existed, when only chemotherapy could be used. In 2005, Grothey et al found that a strategy of making all active agents available to patients with advanced CRC appears to be more important than the use of irinotecan- or oxaliplatin-based combination therapy upfront. ${ }^{23}$ So, we believe that subsequent chemotherapy might not be a determinate factor affecting OS.

In our review, we found that OS was significantly improved by anti-VEGF agent after first-line anti-EGFR therapy. The results revealed that it is a sensible treatment strategy to try anti-VEGF agent after first-line combination therapy with anti-EGFR monoclonal antibody for RAS/ KRAS wild-type mCRC.

\section{Disclosure}

The authors report no conflicts of interest in this work.

\section{References}

1. Grothey A, Sargent D, Goldberg RM, Schmoll HJ. Survival of patients with advanced colorectal cancer improves with the availability of fluorouracil-leucovorin, irinotecan, and oxaliplatin in the course of treatment. J Clin Oncol. 2004;22(7):1209-1214.
2. Herbst RS. Review of epidermal growth factor receptor biology. Int J Radiat Oncol Biol Phys. 2004;59(2 Suppl):21-26.

3. Lin Z, Zhang Q, Luo W. Angiogenesis inhibitors as therapeutic agents in cancer: challenges and future directions. Eur J Pharmacol. 2016; 793:76-81.

4. Zhou M, Yu P, Qu J, et al. Efficacy of bevacizumab in the first-line treatment of patients with ras mutations metastatic colorectal cancer: a systematic review and network meta-analysis. Cell Physiol Biochem. 2016;40(1-2):361-369.

5. Sorich MJ, Wiese MD, Rowland A, Kichenadasse G, McKinnon RA, Karapetis CS. Extended RAS mutations and anti-EGFR monoclonal antibody survival benefit in metastatic colorectal cancer: a meta-analysis of randomized, controlled trials. Ann Oncol. 2015;26(1):13-21.

6. Van Cutsem E, Kohne CH, Lang I, et al. Cetuximab plus irinotecan, fluorouracil, and leucovorin as first-line treatment for metastatic colorectal cancer: updated analysis of overall survival according to tumor KRAS and BRAF mutation status. J Clin Oncol. 2011;29(15):2011-2019.

7. Van Cutsem E, Kohne CH, Hitre E, et al. Cetuximab and chemotherapy as initial treatment for metastatic colorectal cancer. $N$ Engl J Med. 2009;360(14):1408-1417.

8. Maughan TS, Adams RA, Smith CG, et al. Addition of cetuximab to oxaliplatin-based first-line combination chemotherapy for treatment of advanced colorectal cancer: results of the randomised phase $3 \mathrm{MRC}$ COIN trial. Lancet. 2011;377(9783):2103-2114.

9. Bokemeyer C, Bondarenko I, Hartmann JT, et al. Efficacy according to biomarker status of cetuximab plus FOLFOX-4 as first-line treatment for metastatic colorectal cancer: the OPUS study. Ann Oncol. 2011; 22(7):1535-1546

10. Tveit KM, Guren T, Glimelius B, et al. Phase III trial of cetuximab with continuous or intermittent fluorouracil, leucovorin, and oxaliplatin (Nordic FLOX) versus FLOX alone in first-line treatment of metastatic colorectal cancer: the NORDIC-VII study. J Clin Oncol. 2012; 30(15):1755-1762.

11. Douillard JY, Siena S, Cassidy J, et al. Final results from PRIME: randomized phase III study of panitumumab with FOLFOX4 for first-line treatment of metastatic colorectal cancer. Ann Oncol. 2014; 25(7):1346-1355.

12. Heinemann V, von Weikersthal LF, Decker T, et al. FOLFIRI plus cetuximab versus FOLFIRI plus bevacizumab as first-line treatment for patients with metastatic colorectal cancer (FIRE-3): a randomised, open-label, phase 3 trial. Lancet Oncol. 2014;15(10):1065-1075.

13. Moosmann N, von Weikersthal LF, Vehling-Kaiser U, et al. Cetuximab plus capecitabine and irinotecan compared with cetuximab plus capecitabine and oxaliplatin as first-line treatment for patients with metastatic colorectal cancer: AIO KRK-0104 - a randomized trial of the German AIO CRC study group. J Clin Oncol. 2011;29(8):1050-1058.

14. Khattak MA, Martin H, Davidson A, Phillips M. Role of first-line anti-epidermal growth factor receptor therapy compared with antivascular endothelial growth factor therapy in advanced colorectal cancer: a meta-analysis of randomized clinical trials. Clin Colorectal Cancer. 2015;14(2):81-90.

15. Derangere V, Fumet JD, Boidot R, et al. Does bevacizumab impact anti-EGFR therapy efficacy in metastatic colorectal cancer? Oncotarget. 2016;7(8):9309-9321.

16. Venook AP, Niedzwiecki D, Lenz HJ, et al. Effect of first-line chemotherapy combined with cetuximab or bevacizumab on overall survival in patients with kras wild-type advanced or metastatic colorectal cancer: a randomized clinical trial. JAMA. 2017;317(23):2392-2401.

17. Tol J, Koopman M, Cats A, et al. Chemotherapy, bevacizumab, and cetuximab in metastatic colorectal cancer. N Engl J Med. 2009;360(6): 563-572.

18. Brodowicz T, Ciuleanu TE, Radosavljevic D, et al. FOLFOX4 plus cetuximab administered weekly or every second week in the firstline treatment of patients with KRAS wild-type metastatic colorectal cancer: a randomized phase II CECOG study. Ann Oncol. 2013; 24(7):1769-1777. 
19. Hecht JR, Mitchell E, Chidiac T, et al. A randomized phase IIIB trial of chemotherapy, bevacizumab, and panitumumab compared with chemotherapy and bevacizumab alone for metastatic colorectal cancer. J Clin Oncol. 2009;27(5):672-680.

20. Lievre A, Bachet JB, Le Corre D, et al. KRAS mutation status is predictive of response to cetuximab therapy in colorectal cancer. Cancer Res. 2006;66(8):3992-3995.

21. De Roock W, Claes B, Bernasconi D, et al. Effects of KRAS, BRAF, NRAS, and PIK3CA mutations on the efficacy of cetuximab plus chemotherapy in chemotherapy-refractory metastatic colorectal cancer: a retrospective consortium analysis. Lancet Oncol. 2010;11(8):753-762.

22. Benson AB 3rd, Venook AP, Cederquist L, et al. Colon Cancer, Version 1.2017, NCCN Clinical Practice Guidelines in Oncology. J Natl Compr Canc Netw. 2017;15(3):370-398.
23. Grothey A, Sargent D. Overall survival of patients with advanced colorectal cancer correlates with availability of fluorouracil, irinotecan, and oxaliplatin regardless of whether doublet or single-agent therapy is used first line. J Clin Oncol. 2005;23(36):9441-9442.

24. Schwartzberg LS, Rivera F, Karthaus M, et al. PEAK: a randomized, multicenter phase II study of panitumumab plus modified fluorouracil, leucovorin, and oxaliplatin (mFOLFOX6) or bevacizumab plus mFOLFOX6 in patients with previously untreated, unresectable, wild-type KRAS exon 2 metastatic colorectal cancer. J Clin Oncol. 2014;32(21):2240-2247.

25. Qin S, Xu J, Wang L, et al. First-line FOLFOX-4 \pm cetuximab in patients with RAS wild-type (wt) metastatic colorectal cancer (mCRC): the open-label, randomized phase 3 TAILOR trial. Annals of Oncology. 2016;27(suppl 2):141.

\section{Publish your work in this journal}

OncoTargets and Therapy is an international, peer-reviewed, open access journal focusing on the pathological basis of all cancers, potential targets for therapy and treatment protocols employed to improve the management of cancer patients. The journal also focuses on the impact of management programs and new therapeutic agents and protocols on

\section{Dovepress}

patient perspectives such as quality of life, adherence and satisfaction. The manuscript management system is completely online and includes a very quick and fair peer-review system, which is all easy to use. Visit http://www.dovepress.com/testimonials.php to read real quotes from published authors.

\footnotetext{
Submit your manuscript here: http://www.dovepress.com/oncotargets-and-therapy-journal
} 